\title{
Efficacy of the Addition of Prokinetics for Proton Pump Inhibitor (PPI) Resistant Non-erosive Reflux Disease (NERD) Patients: Significance of Frequency Scale for the Symptom of GERD (FSSG) on Decision of Treatment Strategy
}

\author{
Masaki Miyamoto $^{1}$, Noriaki Manabe ${ }^{2}$ and Ken Haruma ${ }^{2}$
}

\begin{abstract}
Background Non-erosive reflux disease (NERD) is a more difficult to treat than reflux esophagitis (RE) due to the high prevalence of PPI resistance. Consequently, the treatment strategy for NERD is yet to be established.

Patients and Methods Subjects were 467 GERD patients (NERD 349, RE 118, $47.4 \pm 16.7$ years) with reflux symptoms such as heartburn. PPI was administered for 2 weeks, and total score (TS) of symptoms, seven items of reflux symptoms e.g. heartburn (reflux score: RS), and five items of dyspeptic symptoms e.g. heavy stomach (dyspeptic score: DS) were assessed using the frequency scale for the symptoms of GERD (FSSG), a GERD-specific questionnaire developed in Japan. Improvement $<50 \%$ in TS was defined as nonresponder. Patients' background, and pretreatment TS, RS, DS, and 12 items of FSSG were assessed. Furthermore, the effect of additional prokinetics (4 weeks) for 117 PPI non-response NERD patients was also examined.

Results Younger age, constipation, higher TS, DS, F2 (bloated stomach), 3 (heavy stomach), 5 (sick feeling after meal), 8 (satiety during meal) in FSSG were factors to be PPI non-responders in NERD. Significant improvement in TS were observed (pretreatment: $17.4 \pm 7.7$ vs. 2 weeks $14.6 \pm 6.0$ vs. 6 weeks $7.7 \pm 5.2, \mathrm{p}<$ 0.0001) after the addition of prokinetics in PPI non-response NERD.

Conclusion Younger age, constipation, dysmotility were factors of PPI non-response in NERD. As high DS is correlated with PPI non-response, it is indicated that patients with strong dysmotility and functional dyspepsia complication might be PPI resistant. The efficacy of additional prokinetics for PPI non-response NERD was observed.
\end{abstract}

Key words: GERD questionnaire, FSSG questionnaire, GERD (gastro-esophageal reflux disease), PPI, prokinetics

(Inter Med 49: 1469-1476, 2010)

(DOI: 10.2169/internalmedicine.49.3615)

\section{Introduction}

Most gastroesophageal reflux disease (GERD) patients with acid reflux into the esophagus are treatable by proton pump inhibitor (PPI). However, non-erosive reflux disease
(NERD) is a difficult-to-treat acid reflux condition even with PPI compared to reflux esophagitis (RE); this is due to the many PPI-resistant cases and various factors other than acid secretion, and the treatment strategy is yet to be established. Recently, the frequency scale for the symptoms of GERD (FSSG), a GERD-specific questionnaire developed in

${ }^{1}$ Department of General Internal Medicine, Prefectural Hiroshima Hospital, Hiroshima and ${ }^{2}$ Division of Gastroenterology, Department of Internal Medicine, Kawasaki Medical School, Kurashiki

Received for publication March 3, 2010; Accepted for publication April 5, 2010

Correspondence to Dr. Masaki Miyamoto, mykmiya@f2.dion.ne.jp 
Table 1. Profile of 467 GERD Patients

\begin{tabular}{ll}
\hline Age $(\mathrm{yrs})$ & $47.4 \pm 16.7$ \\
Gender $(\mathrm{M} / \mathrm{F})$ & $188 / 279$ \\
BMI $\left(\mathrm{kg} / \mathrm{m}^{2}\right)$ & $23.0 \pm 3.6$ \\
H.pylori infection rate $(\%)$ & 25.5 \\
Hiatal hernia (\%) & 20.7 \\
Smoking (\%) & 23.6 \\
Alcohol (\%) & 39.6 \\
Kyphosis (\%) & 3.4 \\
Diabete mellitus $(\%)$ & 2.6 \\
Constipation $(\%)$ & 33.2 \\
\hline
\end{tabular}

Japan (1), has been used for screening GERD patients. The FSSG questionnaire comprises 12 questions concerning not only acid related symptoms, but also dyspeptic (dysmotility) symptoms $(1,2)$. We investigated whether the FSSG questionnaire predicts PPI non-response and determined treatment strategy of NERD in comparison to RE.

\section{Patients and Methods}

The subjects of this study were 467 GERD patients (male/female: 188/279, $47.4 \pm 16.7$ years) with reflux symptoms such as heartburn. Subjects were excluded if they were pregnant, or had a history of gastric surgery, or upper gastrointestinal diseases such as gastric cancer or peptic ulcer. Subjects had initially presented to the Department of General Internal Medicine of Prefectural Hiroshima Hospital complaining of reflux symptoms (heartburn and/or reflux) at least twice weekly. At the time of the initial screening examination, a full medical history was taken and a complete physical examination performed. Blood samples were also taken for measurement of anti-Helicobacter pylori $(\mathrm{Hp})$ antibody (IgG) at enrollment. All 467 subjects were administered the FSSG questionnaire and underwent upper gastrointestinal endoscopy. Endoscopic findings were graded using the Los Angeles (LA) classification (3), with mucosal breaks classified as erosive GERD of LA Grades A-D. Patients with findings not meeting the definition of mucosal breaks in the LA classification, such as those who had prominent erythema without clear demarcation or whitish cloudiness of the lower esophageal mucosa obscuring the longitudinal blood vessels, were classified as having non-erosive reflux disease (NERD) (4). According to modified LA classification, they were divided to 349 NERD patients (male/female: $122 / 227,46.0 \pm 16.5$ years) and $118 \mathrm{RE}$ patients (male/female: $66 / 52,51.6 \pm 16.4$ years). PPI was administered for 2 weeks, and total score (TS) of symptoms, seven items of reflux symptoms e.g. heartburn (reflux score: RS), and five items of dyspeptic symptoms e.g. heavy stomach (dyspeptic score: DS) were assessed using FSSG. Improvement $\geq 50 \%$ in TS was defined as responder while $<50 \%$ was defined as non-responder. Patients' background of responders and nonresponders, pretreatment TS, RS, DS, and 12 items of FSSG were assessed. Furthermore, the effect of additional proki- netics for 117 PPI non-response NERD patients was also examined.

\section{Profile of GERD patients (Table 1)}

Background factors including age, gender, body mass index (BMI), Hp infection, hiatal heria, smoking, alcohol, kyphosis, diabetes mellitus, and symptomatic constipation were examined in all 467 enrolled subjects (Table 1). Constipation was defined in this study as passing stools less than three times a week. Alcohol consumption was defined as drinking more frequently than two days a week.

\section{FSSG questionnaire}

The FSSG questionnaire comprises the following 12 questions: 1) 'Do you feel heartburn?'; 2) 'Does your stomach get bloated?; 3) 'Does your stomach ever feel heavy after meals?'; 4) 'Do you sometimes subconsciously rub your chest with your hand?'; 5) 'Do you ever feel sick after meals?'; 6) 'Do you get heartburn after meals?'; 7) 'Do you have an unusual sensation in your throat?'; 8) 'Do you feel full while eating meals?'; 9) 'Does something become stuck when you swallow?'; 10) 'Does bitter fluid come up into your throat?'; 11) 'Do you burp a lot?'; 12) 'Do you get heartburn when you bend over?'. Subjects answered according to the frequency of their symptoms as follows: never 0 , occasionally 1 , sometimes 2 , often 3 , and always 4 . In the FSSG, there are seven acid reflux-related symptoms (RS: questions $1,4,6,7,9,10$, and 12), five dysmotility-like symptoms (DS: questions 2, 3, 5, 8, and 11) (2). The maximum of total scores (FSSG-TS) is 48 points in FSSG. The FSSG questionnaire was administered pre-treatment and at 2 weeks. FSSG results were considered in terms of total score, reflux score, and dyspeptic score (maximum; TS 48, RS 28, DS 20 points, respectively).

\section{Upper gastrointestinal endoscopy}

Erosive GERD (reflux esophagitis) was diagnosed in 118 cases $(25.3 \%)$, and non-erosive reflux disease (NERD) in 349 cases $(74.7 \%)$. For the 118 cases of erosive GERD, the endoscopic findings according to the LA classification were $\mathrm{A}$ in 82 cases $(69.5 \%), \mathrm{B}$ in $21(17.8 \%), \mathrm{C}$ in $12(10.2 \%)$, and $\mathrm{D}$ in $3(2.5 \%)$.

\section{Protocol}

All 467 patients were administered a PPI for 2 weeks, and total score (TS) of symptoms, seven items of reflux symptoms e.g. heartburn (reflux score: RS), and five items of dyspeptic symptoms e.g. heavy stomach (dyspeptic score: DS) were assessed using the FSSG questionnaire. Before May 2007, we used full dose PPI as initial therapy. After May 2007 (approval of half dose PPI for NERD therapy), we used half dose PPI. Of 467 patients, 365 patients were randomly administered full dose PPI (rabeprazole (RPZ 10 $\mathrm{mg}$ ): 214 cases, lansoprazole (LPZ $30 \mathrm{mg}$ ): 97 cases, omeprazole (OPZ $20 \mathrm{mg}) 54$ case) and 102 patients were administered half dose PPI (LPZ 15 mg: 63 cases, OPZ 10 mg: 39 
cases)). Improvement $\geq 50 \%$ in TS was defined as PPI responder while $<50 \%$ was defined as non-responder. Prokinetics (mosapride citrate $5 \mathrm{mg}$ tid: a selective $5-\mathrm{HT}_{4}$ receptor agonist, stimulates upper gastrointestinal motor activity, and is free of dopamine D2 receptor antagonist properties (5)) were added to PPI for another 4 weeks in PPI non-response NERD patients. PPI dosage for each GERD patient was not changed for 6 weeks. The effect of additional prokinetics for PPI non-response NERD patients was also examined. All subjects gave written informed consent. This prospective study was approved by the Institutional Review Board (IRB) of Prefectural Hiroshima Hospital.

\section{Statistical analysis}

Data are presented as mean \pm SD. Fisher's protected least significant difference (PLSD) test was used for comparisons of rate of PPI non-response between NERD and RE. Fisher's protected least significant difference (PLSD) test was used for comparisons of PPI non-response between PPI full and half dosage both in RE and NERD. p values less than 0.05 were considered significant. Odds ratios (OR) with confidence intervals $(95 \% \mathrm{CI})$ were compared for risk factors of refractoriness to PPI. Statistical analysis was performed using StatView version 5.0 J (SAS Institute Japan, Tokyo, Japan). One-way analysis of variance (ANOVA) and Fisher's PLSD as a post hoc test (StatView, Abacus Concepts Inc., Berkeley, CA, USA) were used to examine changes in FSSG-TS, RS and DS.

\section{Results}

\section{PPI non-response rate (NERD vs RE)}

There was higher tendency of PPI non-response rate in NERD $(52.7 \%, 184 / 349)$ than in $\operatorname{RE~}(42.4 \%, 50 / 118)(\mathrm{p}=$ $0.0516)$.

\section{Factors in PPI non-response (NERD vs RE)}

Younger age, constipation, higher TS, DS, F2 (bloated stomach), 3 (heavy stomach), 5 (sick feeling after meal), 8 (satiety during meal) of FSSG were factors in PPI nonresponder in NERD (Table 2, 3). Younger age, non-kyphosis and higher score in F8 (satiety during meals) of FSSG were factors in PPI non-responder in RE (Table 4, 5). Younger age and higher score in F8 (satiety during meals) of FSSG were factors in PPI non-responder in both NERD and RE.

\section{Addition of prokinetics for PPI-resistant NERD pa- tients}

Significant improvement in TS (pretreatment: $17.4 \pm 7.7$ vs. 2 weeks $14.6 \pm 6.0$ vs. 6 weeks $7.7 \pm 5.2, \mathrm{p}<0.0001)$ was observed after the addition of prokinetics in PPI nonresponse NERD patients (Fig. 1). Significant improvement in DS was observed at 6 weeks despite worsening at 2 weeks ( 2 weeks: $-11.5 \pm 9.0 \%$ vs 6 weeks: $36.7 \pm 7.8 \%$, p< 0.0001) (Fig. 2).

\section{Discussion}

This prospective study demonstrated that younger age, constipation, dysmotilitc symptoms were factors to be PPI non-responder in NERD. As high FSSG-DS correlates with PPI non-response, it is indicated that patients with strong dysmotility and functional dyspepsia (FD) complication might be PPI non-response. This study also demonstrated that the efficacy of additional prokinetics for PPI nonresponse NERD. FSSG questionnaire (GERD specificquestionnaire) could be used on decision of treatment strategy for NERD.

Of the upper gastrointestinal diseases, GERD is an acidrelated disease that remarkably reduces quality of life (QOL) (6). Undoubtedly, a PPI is the first choice regimen for GERD patients (7). PPI therapy cannot completely resolve symptoms in all cases of GERD; Crawley and Schmitt reported that only $58 \%$ of those receiving PPI report a high level of satisfaction (8). In a systematic review of the literature, the PPI symptomatic response pooled rate was $36.1 \%$ in NERD patients and $55.5 \%$ in those with erosive esophagitis (9). Identifying the underlying mechanisms for PPI failure in each individual patient is essential for treatment success (10). The putative mechanisms for refractory GERD include weakly acidic reflux, duodenogastro-esophageal bile reflux, visceral hypersensitivity, delayed gastric emptying, psychological comorbidity, and concomitant functional bowel disorders (11).

It has, however, been difficult until now to identify PPI non-responders prior to treatment. Recently, GERD symptom questionnaires such as the QUEST questionnaire (12), the Gastrointestinal Symptom Rating Scale (GSRS) (13), and the Medical Outcomes Study Short Form-36 health survey (SF36) (14) have come into use for screening GERD and assessing treatment response. Stanghellini et al stated that an ideal GERD symptom questionnaire should possess the following characteristics: sensitivity to patients with GERD; coverage of the frequency and intensity of typical and atypical GERD symptoms; multidimensionality (cover all symptom dimensions); and useful in assessing changes during and after therapy (15). We used the FSSG questionnaire to evaluate GERD patients' reflux symptoms in this study. The FSSG questionnaire is a GERD-specific questionnaire produced by Kusano et al and developed in Japan (1). The FSSG questionnaire comprises 12 questions that cover not only acid symptoms but also dyspeptic (dysmotility) symptoms $(1,2)$. The FSSG provides useful assistance in making the initial diagnosis of GERD, and also allows quantitative assessment of the effects of treatment and changes in symptoms over time $(1,2)$. The FSSG questionnaire has the following characteristics: coverage of the frequency of typical and atypical GERD symptoms; multidimensionality (covers all symptom dimensions); and useful in assessing changes during and after therapy and it is regarded as an ideal GERD symptom questionnaire. In this study, 
Table 2. Background Factors (NERD: PPI Responder vs Non-responder)

\begin{tabular}{cccc}
\hline Factors & $\begin{array}{c}\text { Responder } \\
(\mathrm{n}=165)\end{array}$ & $\begin{array}{c}\text { Non responder } \\
(\mathrm{n}=184)\end{array}$ & p value \\
\hline$\underline{\text { Age }}$ & $48.5 \pm 16.3$ & $43.7 \pm 16.4$ & $\underline{\mathbf{0 . 0 0 6 6}}$ \\
Gender $(\mathrm{M} / \mathrm{F})$ & $61 / 104$ & $61 / 123$ & $\mathrm{NS}$ \\
BMI $\left(\mathrm{kg} / \mathrm{m}^{2}\right)$ & $22.9 \pm 3.7$ & $22.2 \pm 3.6$ & NS \\
H. pylori $(+/-/$ unknown$)$ & $35 / 125 / 5$ & $46 / 132 / 6$ & NS \\
Hiatal hernia $(+/-/$ unknown $)$ & $22 / 111 / 32$ & $16 / 124 / 44$ & NS \\
Smoking $(+/-)$ & $30 / 135$ & $42 / 142$ & NS \\
Alcohol $(+/-)$ & $63 / 102$ & $62 / 122$ & NS \\
Kyphosis $(+/-)$ & $5 / 160$ & $1 / 183$ & NS \\
Diabetes mellitus $(+/-)$ & $4 / 161$ & $4 / 180$ & NS \\
Constipation $(+/-)$ & $41 / 124$ & $76 / 108$ & $\underline{0.0014}$ \\
\hline
\end{tabular}

Table 3. FSSG (NERD: PPI Responder vs Non-responder)

\begin{tabular}{cccc}
\hline Pre FSSG score & $\begin{array}{c}\text { Responder } \\
(\mathrm{n}=165)\end{array}$ & $\begin{array}{c}\text { Non responder } \\
(\mathrm{n}=184)\end{array}$ & p value \\
\hline F1 (Heartburn) & $1.4 \pm 0.1$ & $1.5 \pm 0.1$ & $\mathrm{NS}$ \\
\hline F2 (Broated stomach) & $\underline{1.2 \pm 0.1}$ & $\underline{1.5 \pm 0.1}$ & $\underline{0.0157}$ \\
F3 (Gastric Heaviness after meals) & $\underline{1.4 \pm 0.1}$ & $\underline{1.9 \pm 0.1}$ & $\underline{0.0009}$ \\
\hline F4 (Chest rubbing) & $1.2 \pm 0.1$ & $1.3 \pm 0.1$ & $\mathrm{NS}$ \\
\hline F5 (Sick feeling after meals) & $\underline{1.0 \pm 0.1}$ & $\underline{1.3 \pm 0.1}$ & $\underline{0.0297}$ \\
\hline F6 (Heartburn after meals) & $1.2 \pm 0.1$ & $1.2 \pm 0.1$ & $\mathrm{NS}$ \\
F7 (Burning sensation in throat) & $1.0 \pm 0.1$ & $1.2 \pm 0.1$ & $\mathrm{NS}$ \\
\hline F8(Fullness while meals) & $\underline{0.9 \pm 0.1}$ & $\underline{1.4 \pm 0.1}$ & $\mathbf{0 . 0 0 0 4}$ \\
\hline F9 (Stuckness while swallowing) & $0.7 \pm 0.1$ & $0.8 \pm 0.1$ & $\mathrm{NS}$ \\
F10(Regurgitation into throat) & $1.0 \pm 0.1$ & $1.1 \pm 0.1$ & $\mathrm{NS}$ \\
\hline F11(Burp a lot) & $1.5 \pm 0.1$ & $1.4 \pm 0.1$ & $\mathrm{NS}$ \\
\hline F12 (Heartburn while bending) & $0.5 \pm 0.1$ & $0.7 \pm 0.1$ & $\underline{0.0177}$ \\
\hline FSSG-TS & $\underline{13.3 \pm 0.5}$ & $\underline{15.3 \pm 0.6}$ & $\mathrm{NS}$ \\
FSSG-RS & $7.2 \pm 0.3$ & $7.8 \pm 0.4$ & $\underline{0.0012}$ \\
\hline FSSG-DS & $\underline{6.1 \pm 0.3}$ & $\underline{7.5 \pm 0.3}$ &
\end{tabular}

higher TS, DS, F2 (bloated stomach), 3 (heavy stomach), 5 (sick feeling after meal), 8 (satiety during meal) in FSSG, younger age, and constipation were factors in PPI nonresponders in NERD. Reflux symptoms such as heartburn and reflux are generally regarded as typical symptoms of GERD, although it has been reported that other dyspeptic symptoms are also common in patients with GERD (16). Rome-III committee proposed subdividing FD patients into postprandial distress syndrome (PDS), characterized by postprandial fullness and early satiety, and epigastric pain syndrome (EPS), characterized by epigastric pain and epigastric burning (17).

NERD is a difficult-to-treat acid reflux condition even with PPI compared to reflux esophagitis (RE) due to the many PPI-resistant cases and various factors other than acid secretion, and the treatment strategy is yet to be established. For PPI resistant GERD, doubling the PPI dose or switching to another PPI are pursued by the treating physician (10).
Fass et al reported that doubling the PPI dose could increase the rate of overall symptom improvement by $22-26 \%$ in patients who failed by standard dose PPI therapy (18). However, doubling the PPI dose cannot resolve symptoms in all GERD patients. In this study, there was a significant improvement by standard dose over half dose in RE group $(49.5 \pm 4.0 \%$ vs. $20.3 \pm 13.9 \%, \mathrm{p}<0.05)$, however, there was no difference in efficacy of standard dose and half dose in NERD group $(40.5 \pm 2.5 \%$ vs. $40.0 \pm 4.5 \%$, NS $)$. This result indicated that the doubling dose of PPI might not be optimal for PPI refractory NERD patients. Fass and Sifrim reported that NERD patients tend to have the characteristic of a low esophageal acid exposure profile and lower symptom response to PPI than erosive esophagitis (11). Hiyama et al reported that NERD patients had severe glandular atrophy of the gastric mucosa and frequent resistance to PPI therapy in meta analysis (19). The majority of patients, who present with dysmotility-like symptoms like postprandial 
Table 4. Background Factors (RE: PPI Responder vs Non-responder)

\begin{tabular}{cccc}
\hline Factors & $\begin{array}{c}\text { Responder } \\
(\mathbf{n}=68)\end{array}$ & $\begin{array}{c}\text { Non responder } \\
(\mathbf{n}=50)\end{array}$ & p value \\
\hline Age & $54.4 \pm 16.5$ & $47.7 \pm 15.8$ & $\underline{\mathbf{0 . 0 2 6 1}}$ \\
Gender $(\mathrm{M} / \mathrm{F})$ & $39 / 29$ & $27 / 23$ & NS \\
BMI $\left(\mathrm{kg} / \mathrm{m}^{2}\right)$ & $24.1 \pm 3.4$ & $24.4 \pm 3.2$ & NS \\
H. pylori $(+/-)$ & $22 / 46$ & $14 / 36$ & NS \\
Hiatal hernia $(+/-)$ & $28 / 40$ & $15 / 35$ & NS \\
Smoking $(+/-)$ & $38 / 30$ & $22 / 28$ & NS \\
Alcohol $(+/-)$ & $38 / 30$ & $22 / 28$ & NS \\
Kyphosis $(+/-)$ & $9 / 59$ & $1 / 49$ & $\mathbf{0 . 0 4 2 7}$ \\
Diabetes mellitus $(+/-)$ & $4 / 64$ & $0 / 50$ & NS \\
Constipation $(+/-)$ & $19 / 49$ & $19 / 31$ & NS \\
\hline
\end{tabular}

Table 5. FSSG (RE: PPI Responder vs Non-responder)

\begin{tabular}{cccc}
\hline Pre FSSG score & Responder $(\mathbf{n}=68)$ & $\begin{array}{c}\text { Non responder } \\
(\mathbf{n}=50)\end{array}$ & p value \\
\hline F1 (Heartburn) & $1.9 \pm 0.1$ & $1.6 \pm 0.2$ & NS \\
\hline F2 (Broated stomach) & $1.2 \pm 0.1$ & $1.5 \pm 0.2$ & NS \\
F3 (Gastric Heaviness after meals) & $1.4 \pm 0.1$ & $1.4 \pm 0.2$ & NS \\
\hline F4 (Chest rubbing) & $1.3 \pm 0.1$ & $1.2 \pm 0.2$ & NS \\
\hline F5 (Sick feeling after meals) & $0.9 \pm 0.1$ & $1.0 \pm 0.2$ & NS \\
\hline F6 (Heartburn after meals) & $1.5 \pm 0.1$ & $1.1 \pm 0.2$ & NS \\
F7 (Burning sensation in throat) & $1.0 \pm 0.2$ & $1.1 \pm 0.2$ & NS \\
\hline F8(Fullness while meals) & $0.7 \pm 0.1$ & $1.3 \pm 0.2$ & $\underline{0.0277}$ \\
\hline F9(Stuckness while swallowing) & $0.5 \pm 0.1$ & $0.1 \pm 0.2$ & NS \\
F10(Regurgitation into throat) & $1.5 \pm 0.1$ & $1.4 \pm 0.1$ & NS \\
\hline F11(Burp a lot) & $1.3 \pm 0.1$ & $1.6 \pm 0.2$ & NS \\
\hline F12(Heartburn while bending) & $0.5 \pm 0.1$ & $0.8 \pm 0.2$ & NS \\
\hline FSSG-TS & $13.6 \pm 0.8$ & $15.2 \pm 1.5$ & NS \\
FSSG-RS & $8.2 \pm 0.5$ & $8.2 \pm 0.9$ & NS \\
\hline FSSG-DS & $5.4 \pm 0.4$ & $6.8 \pm 0.7$ & NS \\
\hline
\end{tabular}

fullness or early satiety, do not respond better to acid suppression than to placebo (17). Bredenoord et al discussed that most patients in whom a PPI is not effective do not have GERD, rather they suffer from other disorders such as functional dyspepsia (20). For these patients, drug development has focused on stimulating gastric motility, using gastroprokinetic drugs (17). It has been suggested to use prokinetic therapy as a second-line approach (17). There are several reports describing the efficacy of prokinetics in reflux disease $(21,22)$. Vigneri et al reported that cisapride (prokinetic agent, 5-HT4 receptor agonist) has a synergistic effect with PPIs in maintenance therapy for reflux esophagitis (21). In the USA and Japan, cisapride was withdrawn in 2000 due to an increased risk of cardiological events (severe cardiac arrhythmia) (23). As a replacement for cisapride, mosapride (prokinetic agent, selective $5-\mathrm{HT}_{4}$ receptor agonist) was developed for the symptomatic treatment of patients suffering from various gastrointestinal distur- bances (24). Mosapride decreases the reflux of acid into the esophagus in patients with $\operatorname{GERD}(24,25)$. Madan et al reported that the addition of mosapride is more effective than pantoprazole alone in providing symptomatic relief to patients with erosive GERD, but offers no benefit over pantoprazole alone in non-erosive GERD (22). We previously reported the usefulness of additional prokinetic agents (mosapride) for PPI non-response GERD patients (26). We also reported that cases refractory to PPI therapy showed significant improvement not only in dyspeptic (dysmotility) scores (DS) but also in acid reflux scores (RS) by the addition of a prokinetic agent to the PPI (26). The prokinetic agent (mosapride) may have contributed not only to improved gastrointestinal motility but also to improved pharmacokinetics of the PPI. PPIs are unstable at a low pH. Retention of PPIs inside the stomach for a long time may result in an impaired acid suppressive effect (27), thus rapid transit of the PPI to the upper intestine will be of benefit. 


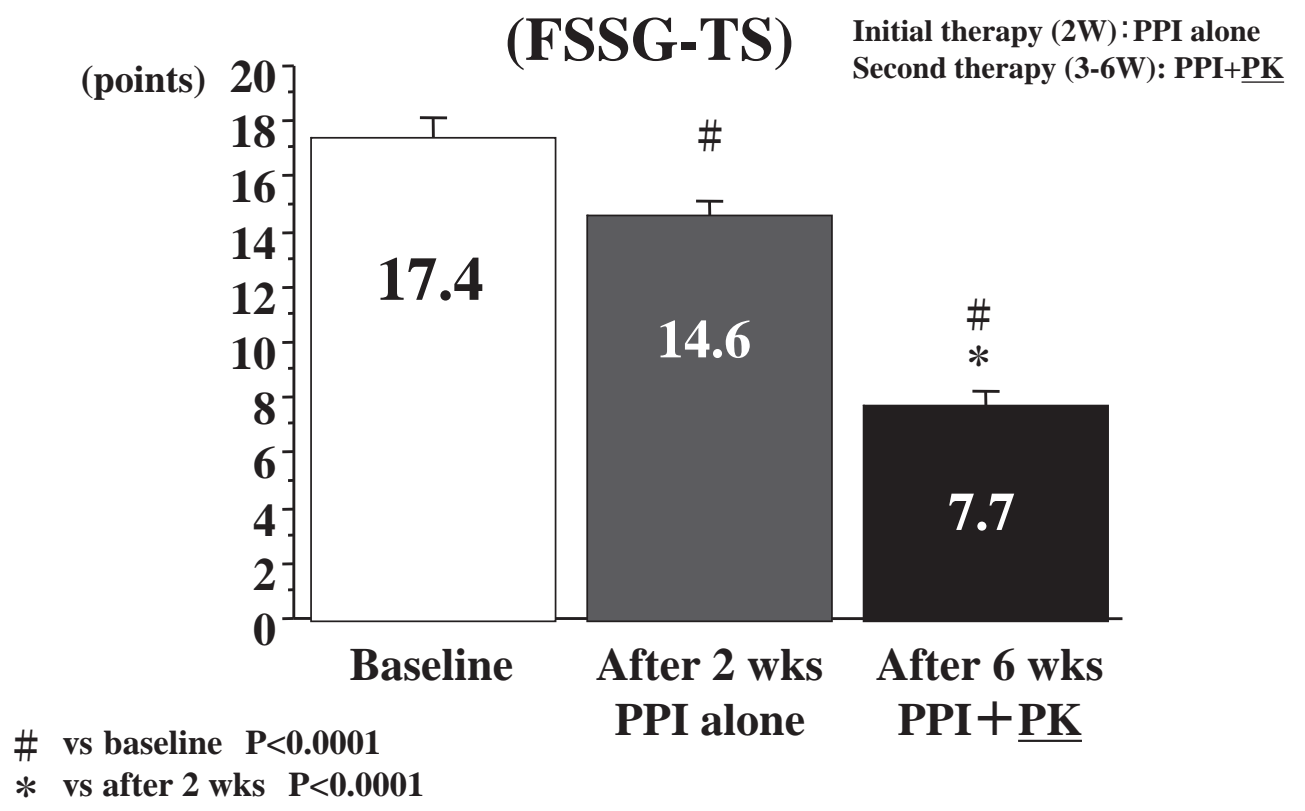

Figure 1. Effect of Prokinetics (PK) with PPI on PPI resistant NERD (FSSG-TS).

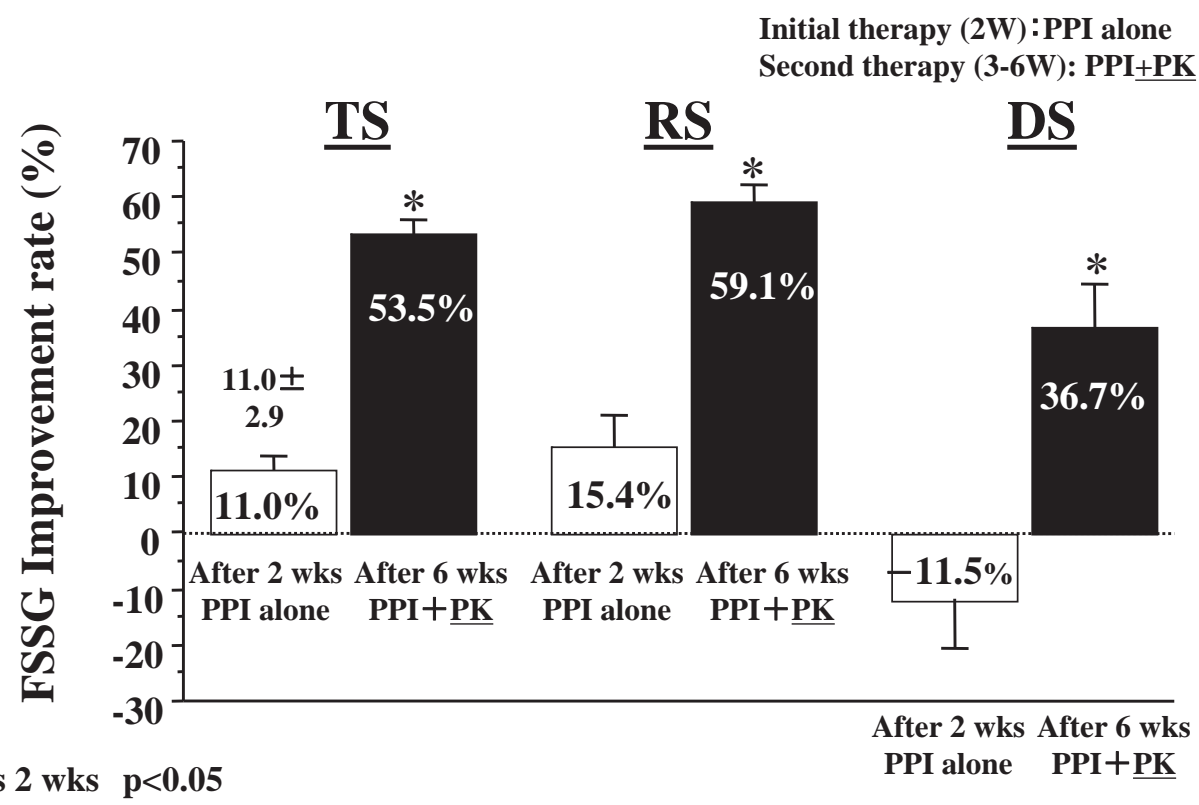

Figure 2. Effect of Prokinetics (PK) with PPI on PPI resistant NERD (FSSG-TS, RS, DS).

Arai et al reported that pharmacokinetic parameters including the mean peak concentration $\left(\mathrm{C}_{\max }\right)$ and the area under the time-concentration curve with 4 hours of dosing $\left(\mathrm{AUC}_{4}\right)$ of the PPI were significantly increased, and the time to reach $\mathrm{C}_{\max }\left(\mathrm{T}_{\max }\right)$ was significantly faster, with the addition of mosapride. They concluded that mosapride, selective $5-\mathrm{HT}_{4}$ receptor agonist, beneficially affects the pharmacokinetics of PPI by accelerating gastric emptying (27). Hiyama et al studied the effect of PPI in NERD patients using MEDLINE. They recommended administration of PPI in combination with a prokinetic agent as first-choice NERD treatment in meta-analysis (19).

The presence of typical dyspeptic symptoms should be assessed by careful history taking and clinical examination.
The use of a simple questionnaire to screen for reflux symptoms may help to identify patterns in patients who actually have reflux disease, with a higher likelihood of these being responders to PPI treatment. The FSSG is useful for detecting not only reflux symptoms such as heartburn and/or reflux, but also dyspeptic (dysmotility) symptoms such as stomach heaviness and/or abdominal distension. The FSSG questionnaire can be used to predict the PPI non-responders in GERD therapy. High pre-treatment FSSG-DS indicates need for the addition of a prokinetic agent to PPI therapy prior to treatment. In this study, younger age was a refractory factor for PPI therapy in NERD. Hiyama et al reported that female gender was associated with PPI resistance in meta-analysis (28). And more, constipation was also a factor 


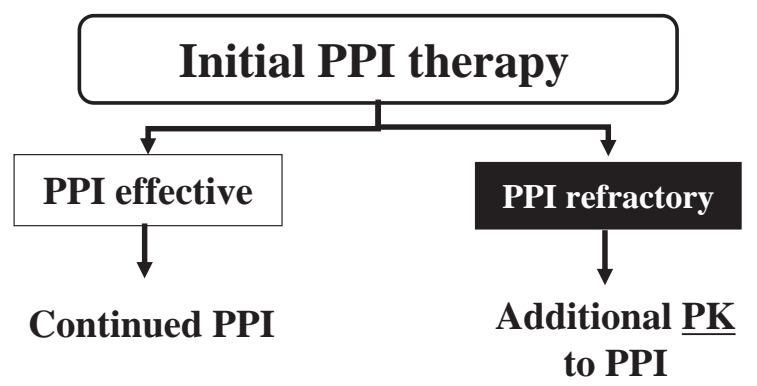

※Recommendation: Additional PK (Prokinetics) to PPI is recommended for NERD patients with strong dysmotility and FD (Functional Dyspepsia).

Figure 3. Strategy for NERD treatment.

in PPI non-responders in NERD. Lin et al reported that many GERD patients also suffer from constipation (29), indicating that they may have reduced motility of the entire gastrointestinal tract. Symptomatic constipation may be a risk factor not only for the occurrence of GERD, but also a refractory factor for PPI therapy. Physicians should mention the symptom of constipation. Most of NERD patients with constipation showed improvement after additional prokinetic agent (mosapride). This pilot study examined whether PPI non-responders were satisfied with additional prokinetic agent. Over two-thirds $(67.5 \%, 79 / 117)$ of PPI non-response NERD patients were satisfied with the addition of prokinetic agent (mosapride) to PPI. Unfortunately, this study lacked a placebo control group who did not receive the addition of prokinetic agent. A further randomized prospective study should be performed.

The results of this study indicated that the symptoms in GERD patients which should be examined include not only acid reflux symptoms, such as heartburn (FSSG-RS) but also dyspeptic symptoms, such as satiety during meal (FSSG-DS). The FSSG questionnaire provides useful information to predict PPI non-responders and determine treatment strategy (e.g. addition of prokinetic to PPI). We recommend an addition of prokinetic agent (mosapride) for PPI non-response NERD (Fig. 3). We anticipate further use of the FSSG questionnaire.

\section{Conclusions}

Younger age, constipation, and dysmotility were factors of PPI non-response in NERD. As high FSSG-DS is correlated to PPI non-response, it is indicated that patients with strong dysmotility and functional dyspepsia complication might be PPI-resistant. The efficacy of additional prokinetic for PPI non-response NERD was observed. FSSG questionnaire (GERD specific-questionnaire) could be used on decision of treatment strategy for NERD.

\section{References}

1. Kusano M, Shimoyama Y, Sugimoto S, et al. Development and evaluation of FSSG: frequency scale for the symptoms of GERD. J Gastroenterol 39: 888-891, 2004.

2. Kusano M, Shimoyama Y, Kawamura O, et al. Proton pump inhibitors improve acid-related dyspepsia in gastroesophageal reflux disease patients. Dig Dis Sci 52: 1673-1677, 2007.

3. Armstrong D, Dent J, Bennett JR, et al. The endoscopic assessment of esophagitis: a progress report on observer agreement. Gastroenterology 111: 85-92, 1996.

4. Hoshihara Y. Reflux esophagitis. In: Atlas of Gastroenterologic Endoscopy by High-Resolution Video-Endoscope. Nagasako K, Fujimori T, Hoshihara Y, Tabuchi M, Eds. Igaku-Shoin, Tokyo, 1998: 32.

5. Yoshida N, Kato S, Ito T. Mosapride Citrate. Drug of the Future 18: 513-515, 1993.

6. Dimenas E. Methodological aspects of evaluation of quality of life in upper gastrointestinal disease. Scand J Gastroenterol 28 (Suppl 199): 18-21, 1993.

7. Dent J, Jones R, Kahrilas P, Talley NJ. Management of gastrooesophageal reflux disease in general practice. BMJ 322: 344-347, 2001.

8. Crawley JA, Schmitt CM. How satisfied are chronic heartburn suffers with their prescription medications? Results of the Patient Unmet Needs Study. J Clin Outcomes Manag 7: 29-34, 2000.

9. Fass R, Shapiro M, Dekel R, Sewell J. Systematic review: protonpump inhibitor failure in gastro-oesophageal reflux disease--where next? Aliment Pharmacol Ther 22: 79-94, 2005 (Review).

10. Fass R. Proton pump inhibitor failure-What are the therapeutic options?. Am J Gastroenterol 104 (Suppl 2): S33-S38, 2005 (Review).

11. Fass R, Sifrim D. Management of heartburn not responding to proton pump inhibitor. Gut 58: 295-309, 2009 (Review).

12. Carlsson R, Dent J, Bolling-Stenevald E, et al. The usefulness of a structured questionnaire in the assessment of symptomatic gastroesophageal reflux disease. Scand J Gastronterol 33: 1023-1029, 1998.

13. Svedlund J, Sjodin I, Dotevall G. GSRS-a clinical rating scale for gastrointestinal symptoms in patients with irritable bowel syndrome and peptic ulcers. Dig Dis Sci 33: 129-134, 1998.

14. Ware JE, Sherbourne CD. The MOS 36-item short form health survey (SF-36). Med Care 30: 473-481, 1992.

15. Stanghellini V, Armstrong D, Monnikes H, Bardhan KD. Systematic review: do we need a new gastro-oesophageal reflux disease questionnaire? Aliment Pharmacol Ther 19: 463-479, 2004.

16. Lee SK, Lee KJ, Kim SJ, Cho SW. Prevalence and risk factors for overlaps between gastroesophageal reflux disease, dyspepsia, and irritable bowel syndrome: a population-based study. Digestion 79: 196-201, 2009.

17. Tack J, Talley NJ, Camilleri M, et al. Functional gastroduodenal disorders. Gastroenterology 130: 1466-1479, 2006 (Review).

18. Fass R, Murthy U, Hayden CW, et al. Omeprazole $40 \mathrm{mg}$ once a day is equally effective as lansoprazole $30 \mathrm{mg}$ twice a day in symptom control of patients with gastro-oesophageal reflux disease (GERD) who are resistant to conventional-dose lansoprazole therapy-a prospective, randomized, multi-centre study. Aliment Pharmacol Ther 14: 1593-1603, 2000.

19. Hiyama T, Yoshihara M, Tanaka S, Haruma K, Chayama K. Strategy for treatment of ninerosive reflux disease in Asia. World $\mathrm{J}$ Gastroenterol 14: 3123-3128, 2008 (Review).

20. Bredenoord AJ, Smout AJ. Refractory gastroesophageal reflux disease. Eur J Gastroenterol Hepatol 20: 217-223, 2008.

21. Vigneri S, Termini R, Leandro G, et al. A comparison of five maintenance therapies for reflux esophagitis. N Engl J Med 333: 1106-1110, 1995.

22. Madan K, Ahuja V, Kashyap PC, Sharma MP. Comparison of efficacy of pantoprazole alone versus pantoprazole plus mosapride in 
therapy of gastroesophageal reflux disease: a randomized trial. Dis Esophagus 17: 274-278, 2004.

23. http://www.fda.gov/bbs/topics/ANSWERS/ANS01007.htm/

24. Ruth M, Finizia C, Cange L, Lundell L. The effect of mosapride on oesophageal motor function in patients with gastro-oesophageal reflux. Eur J Gastroenterol Hepatol 15: 1115-1121, 2003.

25. Ruth M, Hamelin B, Rohss K, Lundell L. The effect of mosapride, a novel prokinetic, on acid reflux variables in patients with gastro-oesophageal reflux disease. Aliment Pharmacol Ther 12: $35-40,1998$.

26. Miyamoto M, Haruma K, Takeuchi K, Kuwabara M. Frequency scale for symptoms of gastroesophageal reflux disease predicts the need for addition of prokinetics to proton pump inhibitor therapy. J Gastroenterol Hepatol 23: 746-751, 2008.

27. Arai K, Takeuchi Y, Watanabe H, Tsukurimichi A, Uchida N, Imawari M. Prokinetics influence the pharmacokinetics of rabeprazole. Digestion 78: 67-71, 2008.

28. Hiyama T, Matsuo K, Urabe Y, et al. Meta-analysis used to identify factors associated with the effectiveness of proton pump inhibitors against non-erosive reflux disease. J Gastroenterol Hepatol 24: 1326-1332, 2009.

29. Lin M, Gerson LB, Lascar R, Davila M, Triadafilopoulos G. Features of gastroesophageal reflux disease in women. Am J Gastroenterol 99: 1442-1447, 2004.

(C) 2010 The Japanese Society of Internal Medicine http://www.naika.or.jp/imindex.html 